\title{
Terapêutica anestésica para o alívio da dor aguda pós-colecistectomia videolaparoscópica: revisão sistemática.
}

\section{Anesthetic therapy for acute pain relief after laparoscopic cholecystectomy: systematic review.}

\author{
Renato Ribeiro de Jesus ${ }^{1}$; Adebaldo Maia Leite ${ }^{1}$; Simone Soares Leite ${ }^{1}$; Márcio Carneiro Vieira ${ }^{1}$; Nivaldo Ribeiro Villela ${ }^{1,2}$
}

R E S U M O

\begin{abstract}
A terapêutica inadequada da dor pós-operatória em colecistectomia videolaparoscópica pode levar a mobilização tardia, insatisfação do paciente, atraso na alta hospitalar e desenvolvimento de dor crônica. Objetivou-se identificar qual a melhor estratégia terapêutica disponível ao anestesiologista na terapia da dor aguda pós-operatória de pacientes submetidos à colecistectomia videolaparoscópica eletiva. Trata-se de revisão sistemática que incluiu 36 artigos completos indexados nas bases de dados Medline, Scopus, Web of Science e LILACS, com recorte temporal de cinco anos (2012 a 2016), resultantes de estudos controlados e randomizados que foram submetidos à análise qualitativa. Em uma proposta de analgesia multimodal, é importante considerar as contraindicações, os efeitos adversos, a dose e o momento ideal das intervenções. Utiliza-se fármacos não opioides, como anti-inflamatórios não esteroides (AINES)/inibidores da ciclo-oxigenase-2 (COX-2), gabapentina/pregabalina, antagonistas dos receptores N-methyl-D-aspartato (NMDA), entre outras. Os opioides podem ser utilizados em doses baixas associadas ou não a terapia multimodal e/ou ficarem restritos aos casos em que a analgesia multimodal não opioide for insuficiente. Conclui-se que não há consenso sobre qual a melhor estratégia analgésica a ser implementada na dor aguda pós-operatória da colecistectomia videolaparoscópica, o que requer sua aplicabilidade de forma individualizada, com base nas evidências científicas encontradas na literatura. Aponta-se como contribuições para o ensino e a prática profissional o enriquecimento teórico das opções medicamentosas analgésicas disponíveis para a terapêutica da dor pós-operatória de pacientes submetidos à colecistectomia videolaparoscópica eletiva, além de alertar a equipe para considerar os efeitos adversos das intervenções implementadas.
\end{abstract}

Descritores: Dor Pós-Operatória. Colecistectomia Laparoscópica. Analgesia. Revisão.

\section{INTRODUÇÃO}

$\mathrm{O}$ tratamento inadequado da dor pós-operatória da colecistectomia videolaparoscópica (CVL) pode levar à consequências negativas, como mobilização tardia com consequente atraso na alta, desenvolvimento de dor crônica e aumento dos custos de tratamento'. Nos últimos anos, tem sido recomendada a analgesia multimodal que combina efeitos aditivos e sinérgicos de diferentes analgésicos com menos efeitos adversos e analgesia mais eficaz ${ }^{1-3}$. Revisões sistemáticas da literatura realizadas em 2006 e em 2011 destacaram intervenções multimodais para aliviar a dor na CVL. Um estudo destacou a administração pré-operatória de dexametasona $8 \mathrm{mg}$ intravenosa (IV), inibidores não esteroides anti-inflamatórios ou da COX-2 e bupivacaína tópica, ouso intraoperatório de antiemético e a administração de bupivacaína intraperitoneal no estabelecimento de pneumoperitônio e drenos ${ }^{4}$. O outro estudo recomendou dose única pré-operatória de dexametasona, anestésicos locais nas incisões (no início ou no final da cirurgia, dependendo da preferência do anestesiologista) e tratamento contínuo com anti-inflamatórios não esteroides (AINES)/inibidores da COX-2, durante os primeiros três a quatro dias, reservando os opioides para quando outras técnicas analgésicas falharem ${ }^{5}$.

Objetivou-se identificar qual a melhor estratégia terapêutica disponível ao anestesiologista na terapia da dor aguda pós-operatória de pacientes submetidos à colecistectomia vídeo-laparoscópica eletiva.

\section{MÉTODOS}

Estudo foi realizado nos meses de junho e julho de 2016, pelo pesquisador principal mais

1 - Universidade Federal do Rio de Janeiro, Departamento de Cirurgia, Serviço de Anestesiologia, Rio de Janeiro, RJ, Brasil. 2 - Universidade do Estado do Rio de Janeiro, Departamento de Cirurgia Geral, Serviço de Anestesiologia, Centro de Ensino e Treinamento em Dor, Rio de Janeiro, RJ, Brasil. 
um revisor independente, seguindo etapas para o delineamento de uma revisão sistemática ${ }^{6}$. Para a busca das produções científicas, utilizaram-se os Descritores em Ciências da Saúde (DeCS) e Medical Subject Headings (MeSH), a partir dos cruzamentos: postoperative pain (dor pós-operatória, dolor postoperatorio) AND laparoscopic cholecystectomy (colecistectomia laparoscópica, colecistectomía laparoscópica) AND analgesia.

Definiram-se como critérios de inclusão os artigos completos indexados nas bases de dados Medical Literature Analysis and Retrieval System Online (Medline), Scopus, Web of Science e Literatura Latino-Americana e do Caribe em Ciências da Saúde (LILACS), compreendidos em um recorte temporal de cinco anos (2012 a 2016), publicados nos idiomas português, inglês ou espanhol, resultantes de estudos controlados e randomizados. Além disso, consideraram-se estudos com adultos/idosos entre 18 e 65 anos de idade, não gestantes, submetidos à colecistectomia videolaparoscópica eletiva. Excluíramse considerações cirúrgicas (instilação de anestésicos locais, quantidade de dióxido de carbono infundido, etc.), colecistectomia aberta, estudos de revisão da literatura e relatos de caso.

Foram encontrados 800 artigos, sendo 136 artigos no Medline, 306 no Scopus, 349 no Web of Science e nove no LILACS. A busca em fontes de dados não publicados, em andamento e literatura cinzenta, foi feita por meio de uma pesquisa na Clinical Trials e no Registro Brasileiro de Ensaios Clínicos. Foram utilizados os descritores "postoperative pain" AND "laparoscopic cholecystectomy" AND "analgesia", tendo sido encontrados 46 artigos na Clinical Trials e nenhum no Registro Brasileiro de Ensaios Clínicos. Após a busca, todas as referências foram enviadas ao EndNote ${ }^{\circledR}$ on-line para organização do estudo e exclusão das duplicatas.

Totalizaram-se 846 artigos, sendo que, deste total, 173 estavam duplicados, restando 673 artigos obtidos na primeira seleção. Em seguida realizou-se a leitura do título e resumo priorizando a sensibilidade em detrimento à especificidade a fim de fazer uma triagem destas referências ${ }^{6}$. Esta foi feita por uma dupla de revisores, de maneira independente. O artigo foi considerado quando pelo menos um dos revisores julgou o artigo elegível. Esse processo resultou em 145 artigos aptos a serem submetidos a uma leitura completa dos estudos. Havendo discordância entre os revisores, estas foram resolvidas ou por consenso entre a dupla ou por consulta a um terceiro revisor.

Houve 109 artigos rejeitados e 36 aceitos para compor o conjunto de estudos definitivos da revisão (Figura 1). Todos os artigos que preencheram os critérios de elegibilidade para a revisão sistemática tiveram sua qualidade metodológica avaliada de forma individual, minimizando-se vieses e maximizando a validade interna e externa ${ }^{6}$. Para isso, foi avaliada a randomização adequada (geração da sequência de alocação), a garantia do sigilo de alocação, o esquema de cegamento (participantes, equipe envolvida na condução do estudo, avaliadores de desfechos), a análise por intenção de tratar, as perdas de seguimentos, bem como, outras fontes de viés, como interrupção precoce do estudo por benefício.

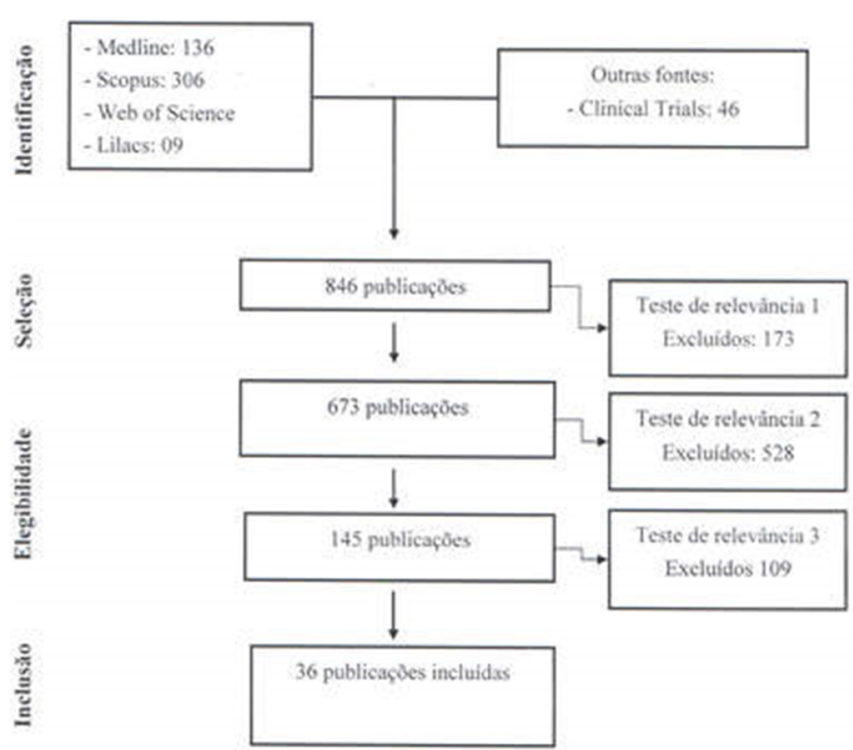

Figura 1. Fluxograma ilustrando a estratégia de busca de artigos. 
Os artigos 1-3,7-38 incluídos nesta revisão sistemática foram organizados e descritos na tabela 1. Mediante a convergência dos achados que respondiam à questão do estudo, agruparam-se as evidências segundo as estratégias terapêuticas disponíveis para o controle da dor aguda pós-CVL eletiva com os comentários necessários à discussão.

\section{RESULTADOS E DISCUSSÃO}

No total, foram identificados 36 ensaios clínicos controlados e randomizados que incluíram 2526 pacientes avaliados (Tabela 1).

\section{AINES/inibidores da COX-2}

Muitos estudos foram feitos avaliando o risco-benefício de diversos AINES/inibidores da COX-2. Os estudos revisados, em sua maioria, apresentaram redução nos escores de dor aguda e na necessidade de opioide no pós-operatório da $C V L$, com efeitos adversos não encontrados e/ou não analisados. Não existe superioridade substancial entre os AINES/inibidores da COX-2 disponíveis, cabendo ao anestesiologista decidir, de acordo com as opções que possui, qual medicamento deseja usar. No entanto, seu uso é recomendado como peça fundamental dentro de uma terapia multimodal,

Tabela 1. Intervenções, avaliação da dor aguda, necessidade de opioide no pós-operatório e efeitos adversos dos estudos incluídos.

\begin{tabular}{|c|c|c|c|c|c|}
\hline \multirow[b]{2}{*}{ Primeiro autor/ano } & \multirow[b]{2}{*}{$\begin{array}{c}\text { № de } \\
\text { pacientes/braços }\end{array}$} & \multirow[b]{2}{*}{ Intervenção } & \multicolumn{2}{|r|}{ Efeitos } & \multirow[b]{2}{*}{$\begin{array}{l}\text { Efeitos } \\
\text { adversos }\end{array}$} \\
\hline & & & $\begin{array}{c}\text { Dor } \\
\text { aguda }\end{array}$ & $\begin{array}{l}\text { Necessidade } \\
\text { de opioides }\end{array}$ & \\
\hline \multicolumn{6}{|l|}{ AINEs/Inibidor da COX } \\
\hline Anil et al., $2016^{1}$ & $60 / 2$ & $\begin{array}{l}\text { Dexketoprofeno trometamol } \\
\text { (vs. diclofenaco) }\end{array}$ & $\neq$ & $\S$ & $\neq$ \\
\hline Fleckenstein et al., $2015^{7}$ & $103 / 2$ & Etoricoxib (vs. placebo) & $\neq$ & $\neq$ & $\neq$ \\
\hline Shuying et al., $2014^{8}$ & $120 / 3$ & $\begin{array}{l}\text { Parecoxib antes da indução } \\
\text { anestésica (vs. parecoxib } \\
\text { após remoção da vesícula } \\
\text { biliar ou vs. placebo) }\end{array}$ & + & $\S$ & $\neq$ \\
\hline Gautam et al., $2014^{9}$ & $120 / 4$ & $\begin{array}{c}\text { Etoricoxib com } \\
\text { metilprednisolona/ } \\
\text { etoricoxib/metilprednisolona } \\
\text { (vs. placebo) }\end{array}$ & + & + & $\ddagger$ \\
\hline Kouroukli et al., $2013^{10}$ & $180 / 3$ & $\begin{array}{l}\text { Lornoxicam/parecoxib } \\
\text { (vs. placebo) }\end{array}$ & + & $\begin{array}{l}\text { ‡ entre as } \\
\text { drogas } / \S \\
\text { vs.placebo }\end{array}$ & $\neq$ \\
\hline Gousheh et al., $2013^{11}$ & $30 / 2$ & Paracetamol (vs. placebo) & + & $\neq$ & - \\
\hline Ureña-Frausto et al., $2013^{12}$ & $56 / 4$ & $\begin{array}{l}\text { Cetoprofeno e tramadol } \\
\text { (vs. cetorolaco) }\end{array}$ & + & $\S$ & $\neq$ \\
\hline Ekmekci et al., $2012^{13}$ & $40 / 2$ & $\begin{array}{l}\text { Tramadol e dexketoprofeno } \\
\text { (vs. tramadol) }\end{array}$ & + & $\S$ & $\neq$ \\
\hline \multicolumn{6}{|l|}{ Antagonista do receptor NMDA } \\
\hline Ozhan et al., $2015^{14}$ & $60 / 2$ & Cetamina (vs. placebo) & $\neq$ & $\S$ & + \\
\hline Yadav et al., $2015^{15}$ & $66 / 2$ & Flupirtina (vs. placebo) & + & $\neq$ & + \\
\hline Kocman et al., $2013^{16}$ & $60 / 3$ & $\begin{array}{l}\text { Sulfato de magnésio } \\
\text { (vs. placebo) }\end{array}$ & + & $\neq$ & $\neq$ \\
\hline Leal et al., 2013'77 & $40 / 2$ & Cetamina (vs. placebo) & $\neq$ & $\neq$ & $\neq$ \\
\hline
\end{tabular}




\begin{tabular}{|c|c|c|c|c|c|}
\hline \multirow[b]{2}{*}{ Primeiro autor/ano } & \multirow{2}{*}{$\begin{array}{c}\text { № de } \\
\text { pacientes/braços }\end{array}$} & \multirow[b]{2}{*}{ Intervenção } & \multicolumn{2}{|c|}{ Efeitos } & \multirow{2}{*}{$\begin{array}{l}\text { Efeitos } \\
\text { adversos }\end{array}$} \\
\hline & & & $\begin{array}{l}\text { Dor } \\
\text { aguda }\end{array}$ & $\begin{array}{l}\text { Necessidade } \\
\text { de opioides }\end{array}$ & \\
\hline Karcioglu et al., $2013^{18}$ & $40 / 2$ & Cetamina (vs. placebo) & + & $\S$ & + \\
\hline Singh et al., $2013^{19}$ & $80 / 4$ & Cetamina (vs. placebo) & + & $\S$ & + \\
\hline Nesek-Adam et al., $2012^{20}$ & $80 / 2$ & $\begin{array}{c}\text { Cetamina e diclofenaco } \\
\text { (vs. placebo) }\end{array}$ & + & $\neq$ & $\neq$ \\
\hline Olgun et al., $2012^{21}$ & $60 / 2$ & Sulfato de Mg (vs. placebo) & + & $\S$ & + \\
\hline \multicolumn{6}{|l|}{ Gabapentina/Pregabalina } \\
\hline Gurunathan et al., $2015^{22}$ & $100 / 4$ & $\begin{array}{c}\text { Pregabalina/celecoxib/ } \\
\text { pregabalina e celecoxib } \\
\text { (vs. placebo) }\end{array}$ & $\neq$ & $\neq$ & + \\
\hline Bekawi et al., 2014²3 & $90 / 3$ & $\begin{array}{c}\text { Pregabalina (vs. } \\
\text { Gabapentina ou vs. placebo) }\end{array}$ & + & $\S$ & + \\
\hline Sarakatsianou et al., $2013^{24}$ & $50 / 3$ & Pregabalina (vs. placebo) & + & $\S$ & + \\
\hline Balaban et al., $2012^{25}$ & $90 / 3$ & Pregabalina (vs. diclofenaco) & + & $\S$ & + \\
\hline \multicolumn{6}{|l|}{ Miscelânea } \\
\hline Bakan et al., 2015² & $80 / 2$ & $\begin{array}{l}\text { Dexmedetomidina e } \\
\text { lidocaína (vs. remifentanil) }\end{array}$ & + & $\neq$ & + \\
\hline Dereli et al., $2015^{26}$ & $60 / 4$ & Esmolol (vs. placebo) & + & $\S$ & + \\
\hline Delfino et al., $2015^{27}$ & $50 / 2$ & Fenilefrina (vs. placebo) & $\neq$ & $\S$ & $\neq$ \\
\hline Akelma et al., $2014^{28}$ & $48 / 3$ & $\begin{array}{l}\text { Esmolol/lidocaína } \\
\text { (vs. remifentamil) }\end{array}$ & + & $\S$ & $\neq$ \\
\hline Ortiz et al., 2014³ & $80 / 4$ & $\begin{array}{l}\text { Propofol (vs. isoflurano vs. } \\
\text { desflurano vs. sevoflurano) }\end{array}$ & $\neq$ & $\neq$ & - \\
\hline Lee et al., $2013^{29}$ & $90 / 3$ & $\begin{array}{l}\text { Nefopam e fentanil } \\
\text { (vs. fentanil) }\end{array}$ & + & $\neq$ & - \\
\hline Park et al., $2012^{30}$ & $42 / 2$ & $\begin{array}{l}\text { Dexmedetomidina } \\
\text { (vs. placebo) }\end{array}$ & + & $\neq$ & - \\
\hline Lopez-Alvarez et al., 201231 & $60 / 2$ & $\begin{array}{l}\text { Esmolol (vs. cetamina e } \\
\text { remifentanil) }\end{array}$ & + & $\S$ & $\neq$ \\
\hline \multicolumn{6}{|l|}{ Bloqueios periféricos } \\
\hline Elamin et al., $2015^{32}$ & $80 / 2$ & $\begin{array}{c}\text { TAP block } \\
\text { (vs. ausência do bloqueio) }\end{array}$ & + & $\neq$ & $\neq$ \\
\hline Basaran, $2015^{33}$ & $76 / 2$ & $\begin{array}{c}\text { TAP block } \\
\text { (vs. ausência do bloqueio) }\end{array}$ & + & $\S$ & $\neq$ \\
\hline Pandey et al., $2015^{34}$ & $60 / 2$ & $\begin{array}{l}\text { Bloqueio epidural com } \\
\text { levobupivacaína e clonidina } \\
\text { (vs. ropivacaína e clonidina) }\end{array}$ & + & $\neq$ & + \\
\hline Bathia et al., 20145 & $60 / 3$ & $\begin{array}{l}\text { TAP block subcostal } \\
\text { (vs.TAP block posterior) }\end{array}$ & + & $\S$ & $\neq$ \\
\hline Petersen et al., $2012^{36}$ & $80 / 2$ & $\begin{array}{l}\text { TAP block com ropivacaína } \\
\text { (vs. TAP block placebo) }\end{array}$ & + & $\S$ & $\neq$ \\
\hline \multicolumn{6}{|l|}{ Opioides } \\
\hline Choi et al., 201537 & $54 / 2$ & Oxicodona (vs. fentanil) & + & $\neq$ & $\neq$ \\
\hline Hwang et al., $2014^{38}$ & $81 / 2$ & Oxicodona (vs. fentanil) & + & $\neq$ & + \\
\hline
\end{tabular}

Legenda: - Não investigado; + Efeito significante no grupo tratado; † Efeito não significante no grupo tratado; § Redução de opioides no pós-operatório. Estudos de grau de recomendação $A$ - nível de evidência $1 B$. 
principalmente por seus benefícios analgésicos e pela raridade e pouca importância de seus efeitos adversos. Algumas opções são apresentadas no texto e servem como embasamento científico para tomada de conduta.

O parecoxib 40mg intravenoso (IV) utilizado 30-45 minutos antes da indução anestésica foi associado a menor dor e consumo de opioides pósoperatórios, quando comparado à sua administração após a remoção da vesícula biliar ou com o placebo ${ }^{\text {. }}$ O uso de lornoxicam $8 \mathrm{mg}$ via oral (VO) mostrou eficácia analgésica semelhante ao parecoxib 40mg IV e melhor do que o placebo, quando administrado em três tomadas (30 minutos antes da cirurgia, 12 e 24 horas após). A necessidade de opioides no pósoperatório foi semelhante entre o lornoxicam e o parecoxib ${ }^{10}$.

A administração associada ou não de metilprednisolona 125mg IV e etoricoxib 120mg VO uma hora antes da cirurgia foi mais eficaz na redução do consumo de opioides, sem que houvesse maior incidência de efeitos adversos. Porém apenas o uso associado mostrou melhor escores de dor

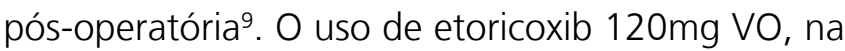
manhã da cirurgia e por três dias seguintes a ela, não mostrou superioridade ao placebo na redução da dose cumulativa de opioide após CVL ou nos escores de dor perioperatória 7 .

O uso de cetorolaco $1 \mathrm{mg} / \mathrm{kg}$ IV em bolus na indução mostrou piores escores de dor aguda e maior necessidade de opioides de resgate em comparação aos grupos nos quais foi administrado fármacos 60 minutos antes da cirurgia em infusão contínua. Esses grupos foram cetoprofeno 100mg IV seguido de 2mg/ kg/24h; cetoprofeno 50mg IV associado à tramadol $50 \mathrm{mg} \mathrm{IV}$, seguidos de $1 \mathrm{mg} / \mathrm{kg} / 24 \mathrm{~h}$ e $0,1 \mathrm{mg} / \mathrm{kg} / \mathrm{h}$, respectivamente, além de um grupo utilizando o dobro da dose de ataque e manutenção do grupo anterior. Este último grupo que recebeu cetoprofeno $100 \mathrm{mg}$ IV associado a tramadol 100mg IV com manutenção de $2 \mathrm{mg} / \mathrm{kg} / 24 \mathrm{~h}$ e $0,2 \mathrm{mg} / \mathrm{kg} / \mathrm{h}$, respectivamente, obteve menor necessidade de opioides no pós-operatório em relação aos demais grupos ${ }^{12}$.

A administração de dose única de dexcetoprofeno trometamol 50mg IV 30 minutos antes do final da cirurgia apresentou escores de dor pós-operatória semelhantes ao uso de diclofenaco sódico $75 \mathrm{mg}$ IV em pacientes submetidos à CVL. Porém, o consumo de opioide foi maior no grupo dexcetoprofeno trometamol comparado com o grupo diclofenaco de sódico. Não se notou aumento dos efeitos adversos ${ }^{1}$. O uso de dexcetoprofeno trometamol 100mg IV associado ao tramadol $600 \mathrm{mg}$ IV, via analgesia controlada pelo paciente, quando utilizado em solução fisiológica $0,9 \%$ de $100 \mathrm{ml}$ com bolus de $3 \mathrm{ml}$, intervalo entre as doses de 15 minutos e $15 \mathrm{ml}$ em quatro horas como limite, mostrou menor consumo de analgésico no pósoperatório sem que houvesse aumento dos efeitos adversos quando comparado ao uso isolado de tramadol 600mg IV ${ }^{13}$.

A administração de paracetamol $1 \mathrm{~g}$ IV imediatamente após a indução da anestesia reduziu a dor de forma significativa em relação ao placebo, porém sem mostrar redução no consumo de opioide após cirurgias de $\mathrm{CVL}^{11}$.

\section{Antagonistas do receptor NMDA (N-metil-D-aspartato)}

Em relação aos antagonistas do receptor NMDA, grande parte dos estudos trouxeram menores escores de dor aguda e necessidade de opioide no pós-operatório da CVL, contudo apontaram efeitos adversos significativos, dependendo da intervenção utilizada. O anestesiologista deverá levar isso em consideração quando optar por seu uso dentro da terapia multimodal, uma vez que influenciará a dinâmica anestésica e do perioperatótio.

O uso de cetamina $1 \mathrm{mg} / \mathrm{kg}$ IV em bolus na indução, seguido de $25 \mathrm{mcg} / \mathrm{kg} / \mathrm{min}$ IV até o fim da cirurgia, utilizada como forma suplementar ao propofol e alfentanil na anestesia venosa total, produz menores escores de dor pós-operatória além 
de diminuir o consumo de opioide em comparação com o placebo. Contudo houve maior tempo até a extubação e alta da sala de recuperação pósanestésica ${ }^{18}$. Quando administrada na dose de 5mcg/ kg/min IV, com alteração da sua dosagem conforme parâmetros hemodinâmicos, no momento da indução até o final da cirurgia, não alterou a intensidade da dor pós-operatória e a necessidade de opioide no pós-operatório de $\mathrm{CVL}^{17}$. Na dose de 0,25mg/kg IV em bolus dois minutos antes da indução, reduziu os escores de dor aguda e necessidade de opioides no pós-operatório em relação ao placebo. Porém, os escores do Aldrete se mostraram inferiores, observando-se maior sedação pelos escores de Ramsay, além de prejuízos na resposta verbal e orientação após a extubação ${ }^{14}$.

A cetamina na dose de 0,5-1 mg/kg IV administrada 30 minutos antes da indução foi eficaz no controle da dor aguda e necessidade de opioide no pós-operatório de CVL se comparada com placebo. Como efeito colateral notou-se aumento da frequência cardíaca (FC) e presença de alucinações ${ }^{19}$. $\mathrm{Na}$ dose de $0,15 \mathrm{mg} / \mathrm{kg}$ antes da incisão cirúrgica, associada a diclofenaco sódico 1mg/kg IV 20 minutos antes da indução da anestesia, mostrou reduzir os escores de dor no pós-operatório, mas não o consumo de opioide. Porém, o uso individual da cetamina não mostrou esses achados e o uso isolado do diclofenaco sódico não poupou opioide no pós-operatório ${ }^{20}$.

A administração de sulfato de magnésio 40mg/kg IV antes da indução, seguido de 10mg/ $\mathrm{kg} / \mathrm{h}$ no intraoperatório, mostrou menores escores de dor e requerimento de opioide no pós-operatório. No entanto levou à respiração inadequada e a um atraso na recuperação, conforme demonstrado por um escore de Aldrete diminuído ${ }^{21}$. Sua administração antes da indução anestésica de 5,0mg/kg e 7,5mg/kg reduziu a dor pós-operatória aguda após a CVL se comparada com placebo, contudo verificou-se que $7,5 \mathrm{mg} / \mathrm{kg}$ foram mais eficazes. Não houve diferença quanto aos efeitos adversos e ao consumo de opioide no pós-operatório ${ }^{16}$.
O uso de flupirtina 200mg vO duas horas antes da cirurgia mostrou-se superior ao placebo no que se refere à dor aguda pós-operatória, porém não houve diferença quanto ao consumo de opioide. No entanto, houve maior incidência de sedação no grupo estudado ${ }^{15}$.

\section{Gabapentina/Pregabalina}

Em geral, o uso de gabapentina/pregabalina em pacientes submetidos à CVL também mostrou menores escores de dor aguda e necessidade de opioide no pós-operatório, porém diversos estudos mostraram efeitos adversos que podem limitar seu uso, como sedação, atraso na extubação e tontura. A administração de pregabalina 150mg ou 300mg VO uma hora antes da cirurgia comparada com placebo diminuiu a intensidade da dor e o consumo de opioides após CVL. Notou-se maior grau de sedação na escala de Ramsay no grupo que recebeu pregabalina $300 \mathrm{mg}^{25}$. Essa dosagem, na noite anterior à cirurgia e uma hora antes da cirurgia, se comparada com placebo, também obteve estes resultados positivos no que tange à analgesia, embora associados ao atraso no tempo de extubação, bem como, ao aumento da incidência de tontura pós-operatória ${ }^{24}$.

O uso isolado de pregabalina 150mg vO ou em combinação com celecoxib 400mg uma hora antes da cirurgia não proporcionou menores escores de dor ou necessidade de opioide quando comparado ao placebo. O grupo que recebeu pregabalina apresentou mais tontura e sonolência ${ }^{22}$. O uso de gabapentina 1200mg VO duas horas antes da cirurgia, seguido da mesma dose 12 horas do pós-operatório e a cada oito horas por dois dias e pregabalina 150mg VO no mesmo esquema de administração, reduziram a necessidade de opioides em pacientes submetidos a CVL, quando comparado com o uso de placebo. O grupo pregabalina mostrou menores escores de dor quando comparado com os demais grupos. As intervenções não placebo resultaram em maior grau de tontura e sonolência ${ }^{23}$. 


\section{Miscelânea}

As intervenções que não puderam ser reunidas nos grupos anteriores foram consideradas miscelânea. Essas mostraram resultados satisfatórios no combate à dor aguda e necessidade de opioide no pós-operatório da CVL, todavia vários de seus efeitos adversos não foram ressaltados nos estudos ou não foram avaliados. O anestesiologista ao conduzir o caso deverá sempre considerar o riscobenefício de suas intervenções.

A infusão de dexmedetomidina $1 \mathrm{mg} / \mathrm{kg} /$ min IV antes da indução, seguida de $0,5 \mathrm{mcg} / \mathrm{kg} / \mathrm{h}$, mostrou redução nos escores de dor pós-operatória apenas na primeira hora se comparado ao placebo. Não houve diferença quanto à necessidade de opioide no pós-operatório. O grupo com a intervenção mostrou menores valores de índice bispectral (BIS), pressão arterial sistólica (PAS) e FC ${ }^{30}$.

A anestesia venosa total livre de opioides com propofol, dexmedetomidina 0,6 mcg/kg IV seguida de 0,3mcg/ $/ \mathrm{kg} / \mathrm{h}$ e lidocaína $1,5 \mathrm{mg} / \mathrm{kg}$ IV e de $2 \mathrm{mg} / \mathrm{kg} / \mathrm{h}$ no intraoperatório está associada ao menor consumo de opioide e escores de dor no pós-operatório em comparação com a anestesia venosa total com remifentanil e propofol. Observaram-se maiores eventos hipertensivos e tempo de recuperação mais prolongado no grupo sem opioide².

O uso de esmolol 1 mg/kg seguido de 10mcg/ kg/min IV após a indução da anestesia até o final da cirurgia, seja associado ao propofol e remifentanil ou ao desflurano e remifentanil, diminuiu a necessidade de opioide e dor no pós-operatório quando comparado ao não uso. Como efeitos adversos notou-se maior redução da FC, mas a pressão arterial média manteve-se a mesma ${ }^{26}$. O esmolol 0,5mcg/kg IV, quando usado na indução seguido de 5-15mcg/ $\mathrm{kg} / \mathrm{min}$ até o fim da cirurgia, reduziu a necessidade de opioide e melhorou a analgesia pós-operatória quando comparado ao grupo que recebeu cetamina 0,5mg/kg IV na indução ${ }^{31}$.
A infusão de esmolol $1 \mathrm{mg} / \mathrm{kg}$ IV antes da indução seguida de $50 \mathrm{mcg} / \mathrm{kg} / \mathrm{min}$ até o fim da cirurgia e/ou lidocaína $2 \mathrm{mg} / \mathrm{kg} / \mathrm{min}$, seguida de $2 \mathrm{mg} / \mathrm{kg} / \mathrm{min}$ na mesma maneira, mostrou menor consumo de opioide na sala de recuperação pós-anestésica. O uso do esmolol resultou em diminuição do consumo de opioides estendendo-se até 24 horas do pós-operatório. Não houve redução nos escores de dor no pós-operatório ${ }^{28}$.

O uso de fenilefrina no intraoperatório a fim de manter a PAS 20-30\% acima do valor basal reduziu o consumo de opioide e os escores de dor após a CVL quando comparado à manutenção da PAS 20-30\% abaixo do valor basal. Não houve variação nos parâmetros hemodinâmicos pósoperatórios. Nesse estudo, segundo o autor não foi possível distinguir se esse efeito analgésico advinha do aumento da PAS ou resultado do efeito direto da fenilefrina como moduladora da dor ${ }^{27}$.

A administração de nefopam $20 \mathrm{mg}$ ou 40mg IV com fentanil 50 ug IV no final da cirurgia reduziu a dor pós-operatória e os efeitos colaterais do opioide quando comparada ao uso isolado de fentanil. Não houve avaliação da necessidade de opioide no pósoperatório $^{29}$. A manutenção da anestesia geral com propofol, em comparação com isoflurano, desflurano ou sevoflurano, não resultou em escores menores de dor e consumo de opioide após CVL. Não foram avaliados efeitos adversos das medicações utilizadas ${ }^{27}$.

\section{Boqueios periféricos}

Na maioria dos estudos, o emprego dos bloqueios periféricos na analgesia dos pacientes submetidos à CVL reduziu os escores de dor aguda e necessidade de opioide no pós-operatório. No entanto, alguns estudos desencorajaram seu uso rotineiro devido aos efeitos adversos, que, apesar de raros, não justificam seu uso neste tipo de cirurgia. Cabe ao profissional anestesiologista conhecer as opções anestésicas disponíveis no hospital em questão e possíveis a ser usadas no paciente para montar seu arsenal analgésico. 
Pacientes que receberam o bloqueio do plano transverso abdominal (TAP block) guiado por ultrassonografia após CVL tiveram escores de dor reduzidos quando tossindo, mas não em repouso, quando comparados com os que não receberam o bloqueio. O consumo de opioide pós-operatório foi menor ${ }^{36}$. Outro estudo semelhante mostrou redução da dor aguda no pós-operatório, porém não foi avaliada a redução da necessidade de opioides no pós-operatório ${ }^{32}$.

Uma abordagem subcostal oblíqua no uso do TAP block com injeção única foi eficaz no controle da dor no pós-operatório de CVL e diminuiu o consumo de opioide ${ }^{33}$. Esse bloqueio mostrou menores escores de dor e necessidade de opioide no pós-operatório em comparação com o bloqueio TAP posterior ou ausência de bloqueio ${ }^{35}$. Quando foram comparados dois grupos submetidos a bloqueio epidural numa cirurgia de CVL, o uso associado de clonidina com levobupivacaína resultou em menor necessidade analgésica no tratamento de dor no ombro em comparação à clonidina com ropivacaína, porém ambos os grupos apresentaram um alto declínio na pressão arterial, necessitando vasopressores ${ }^{34}$.

\section{Opioides}

Os fármacos opioides são a melhor opção no que se refere à analgesia. Contudo, devido ao seu potencial de dependência química e seus efeitos adversos, diversos outros medicamentos surgem no mercado todos os dias. Atualmente, recomenda-se a combinação de fármacos a fim de se aproximar da qualidade analgésica dos opioides. Terapia conhecida como multimodal. As pesquisas na literatura envolvendo opioides objetivam maior eficácia com os menores efeitos adversos possíveis. Nesse sentido, o uso de oxicodona vem se mostrando promissor em pacientes submetidos à $C V L^{37}$.
O uso de oxicodona $0,08 \mathrm{mg} / \mathrm{kg}$ IV 20 minutos antes do final da cirurgia aliviou a dor pós-operatória imediata quando comparado com o de fentanil $1 \mathrm{mcg} / \mathrm{kg}$, não sendo associado a um aumento nos efeitos colaterais em pacientes submetidos à CVL. A necessidade de doses adicionais foi semelhante entre os grupos ${ }^{37}$. Com relação ao seu uso via analgesia controlada pelo paciente, a oxicodona $1 \mathrm{mg}$ IV por bolus mostrou efeitos semelhantes para o alívio da dor em comparação com o fentanil $10 \mathrm{mcg} \mathrm{IV}$, porém apresentou maior incidência de náuseas e vômitos pós-operatórios ${ }^{38}$.

Apesar da viabilidade das várias estratégias para o alívio da dor aguda de pacientes submetidos a CVL, alguns estudos não investigaram os efeitos adversos das intervenções, apenas a eficácia quanto à analgesia pós-operatória e à necessidade de opioide. Isso pode ser explicado pela raridade de suas ocorrências, dificuldade de avaliação ou por não ser objetivo desses estudos ${ }^{4}$.

A heterogeneidade dos estudos da revisão pode ter possibilitado fragilidades na discussão, contudo, esta revisão sistemática da literatura trouxe como contribuições para o ensino e a prática profissional o enriquecimento teórico das ferramentas analgésicas disponíveis para a terapêutica da CVL, além de alertar a equipe para considerar os efeitos adversos das intervenções implementadas. Nosso estudo não considerou as estratégias cirúrgicas na terapêutica do alívio da dor aguda de pacientes submetidos a CVL, porém essas devem ser valorizadas na busca por uma melhor estratégia analgésica.

\section{CONCLUSÃO}

Não há consenso sobre qual a melhor estratégia analgésica a ser implementada na dor aguda pós-operatória da colecistectomia vídeolaparoscópica, o que requer sua aplicabilidade de forma individualizada, com base nas evidências científicas encontradas na literatura. 


\title{
A B S T R A C T
}

\begin{abstract}
Inappropriate therapy of postoperative pain in laparoscopic cholecystectomy may lead to late mobilization, patient dissatisfaction, delayed hospital discharge, and chronic pain development. Our objective was to identify the best therapeutic strategy available to the anesthesiologist for the acute postoperative pain of patients submitted to elective laparoscopic cholecystectomy. This is a systematic review that included 36 complete articles indexed in the Medline, Scopus, Web of Science and LILACS databases, with a five-year time cut (2012 to 2016), resulting from controlled and randomized studies that were submitted to qualitative analysis. In a proposal for multimodal analgesia, it is important to consider the contraindications, adverse effects, dose and optimal timing of interventions. Non-opioid drugs, such as non-steroidal anti-inflammatory drugs (NSAIDs)/cyclooxygenase-2 (COX-2) inhibitors, gabapentin/pregabalin, N-methyl$D$-aspartate (NMDA) receptor antagonists, and others. Opioids may be used at low doses associated with multimodal therapy or are restricted to cases where non-opioid multimodal analgesia is insufficient. We conclude that there is no consensus as to the best analgesic strategy to be implemented in the acute postoperative pain of laparoscopic cholecystectomy, which requires its applicability in an individualized way, based on the scientific evidence found in the literature. As contribution to medical learning and practice, we point out the theoretical enrichment of the analgesic drug options available for the therapy of postoperative pain in patients submitted to elective laparoscopic cholecystectomy, and alert the team to consider the adverse effects of the interventions implemented.
\end{abstract}

Keywords: Pain, Postoperative. Cholecystectomy, Laparoscopic. Analgesia. Review.

\section{REFERÊNCIAS}

1. Anil A, Kaya FN, Yavascaoglu B, Mercanoğlu Efe E, Türker G, Demirci A. Comparison of postoperative analgesic efficacy of intraoperative single-dose intravenous administration of dexketoprofen trometamol and diclofenac sodium in laparoscopic cholecystectomy. J Clin Anesth. 2016;32:127-33.

2. Bakan M, Umutoglu T, Topuz U, Uysal H, Bayram $\mathrm{M}$, Kadioglu $\mathrm{H}$, et al. Opioid-free total intravenous anesthesia with propofol, dexmedetomidine and lidocaine infusions for laparoscopic cholecystectomy: a prospective, randomized, double-blinded study. Rev Bras Anestesiol. 2015;65(3):191-9. Portuguese.

3. Ortiz J, Chang LC, Tolpin DA, Minard CG, Scott $B G$, Rivers JM. Randomized, controlled trial comparing the effects of anesthesia with propofol, isoflurane, desflurane and sevoflurane on pain after laparoscopic cholecystectomy. Rev Braz Anesthesiol. 2014;64(3):145-51.

4. Ahn Y, Woods J, Connor S. A systematic review of interventions to facilitate ambulatory laparoscopic cholecystectomy. HPB (Oxford). 2011;13(10):67786.

5. Bisgaard T. Analgesic treatment after laparoscopic cholecystectomy: a critical assessment of the evidence. Anesthesiology. 2006;104(4):835-46.

6. Galvão TF, Pansani TSA, Harrad D. Principais itens para relatar revisões sistemáticas e meta-análises: a recomendação PRISMA. Epidemiol Serv Saúde. 2015;24(2):335-42.
7. Fleckenstein J, Kohls N, Evtouchenko E, Lehmeyer L, Kramer S, Lang PM, et al. No effect of the cyclooxygenase-2 inhibitor etoricoxib on preemptive and post-operative analgesia in visceral surgery: results of a randomized controlled trial. Eur J Pain. 2016;20(2):186-95.

8. Shuying $L$, Xiao W, Peng L, Tao Z, Ziying L, Liang Z . Preoperative intravenous parecoxib reduces length of stay on ambulatory laparoscopic cholecystectomy. Int J Surg. 2014;12(5):464-8.

9. Gautam S, Agarwal A, Das PK, Agarwal A, Kumar S, Khuba S. Evaluation of the efficacy of methylprednisolone, etoricoxib and a combination of the two substances to attenuate postoperative pain and PONV in patients undergoing laparoscopic cholecystectomy: a prospective, randomized, placebocontrolled trial. Korean J Pain. 2014;27(3):278-84.

10. Kouroukli I, Zompolas V, Tsekoura V, Papazoglou I, Louizos A, Panaretou V. Comparison between lornoxicam quick-release and parecoxib for postoperative analgesia after laparoscopic cholecystectomy: a prospective randomized, placebo-controlled trial. J Anaesthesiol Clin Pharmacol. 2013;29(4):485-90.

11. Gousheh SM, Nesioonpour S, Javaher Foroosh F, Akhondzadeh R, Sahafi SA, Alizadeh Z. Intravenous paracetamol for postoperative analgesia in laparoscopic cholecystectomy. Anesth Pain Med. 2013;3(1):214-8.

12. Ureña-Frausto CA, Plancarte-Sánchez R, ReyesTorres Jl, Ramírez-Aranda JM. Infusional therapy: an alternative for shouder pain post-laparoscopy. Cir Cir. 2013;81(3):187-95. Spanish. 
13. Ekmekçi P, Kazak Bengisun Z, Kazbek BK, Özis $\mathrm{SE}$, Tastan $\mathrm{H}$, Süer AH. The efficacy of adding dexketoprofen trometamol to tramadol with patient controlled analgesia technique in postlaparoscopic cholecystectomy pain treatment. Agri. 2012;24(2):63-8.

14. Ozhan Y, Bakan N, Karaoren GY, Tomruk SG, Topaç Z. Effects of subanesthetic ketamine on pain and cognitive functions in TIVA. J Clin Anal Med. 2015;6(4):452-7.

15. Yadav G, Behera SS, Das SK, Jain G, Choupoo S, Raj J. Role of flupirtine as a preemptive analgesic in patients undergoing laparoscopic cholecystectomy. J Anaesthesiol Clin Pharmacol. 2015;31(2):169-73.

16. Kocman IB, Krobot R, Premuzić J, Kocman I, Stare R, Katalinić $L$, et al. The effect of preemptive intravenous low-dose magnesium sulfate on early postoperative pain after laparoscopic cholecystectomy. Acta Clin Croat. 2013;52(3):289-94.

17. Leal PC, Sakata RK, Salomão R, Sadatsune EJ, Issy AM. Assessment of the effect of ketamine in combination with remifentanil on postoperative pain. Braz J Anesthesiol. 2013;63(2):178-82.

18. Karcioglu M, Davarci I, Tuzcu K, Bozdogan YB, Turhanoglu S, Aydogan A, et al. Addition of ketamine to propofol-alfentanil anesthesia may reduce postoperative pain in laparoscopic cholecystectomy. Surg Laparosc Endosc Percutan Tech. 2013;23(2):197-202.

19. Singh $H$, Kundra $S$, Singh RM, Grewal A, Kaul TK, Sood D. Preemptive analgesia with Ketamine for Laparoscopic cholecystectomy. J Anaesthesiol Clin Pharmacol. 2013;29(4):478-84.

20. Nesek-Adam V, Grizelj-Stojčić E, Mršić V, Rašić $Z$, Schwarz D. Preemptive use of diclofenac in combination with ketamine in patients undergoing laparoscopic cholecystectomy: a randomized, double-blind, placebo-controlled study. Surg Laparosc Endosc Percutan Tech. 2012;22(3):232-8.

21. Olgun B, Oguz G, Kaya M, Savli S, Eskiçirak $H E$, Güney I, et al. The effects of magnesium sulphate on desflurane requirement, early recovery and postoperative analgesia in laparascopic cholecystectomy. Magnes Res. 2012;25(2):72-8.
22. Gurunathan U, Rapchuk IL, King G, Barnett AG, Fraser JF. The effect of pregabalin and celecoxib on the analgesic requirements after laparoscopic cholecystectomy: a randomized controlled trial. J Anesth. 2016;30(1):64-71.

23. Bekawi MS, El Wakeel LM, Al Taher WM, Mageed WM. Clinical study evaluating pregabalin efficacy and tolerability for pain management in patients undergoing laparoscopic cholecystectomy. Clin J Pain. 2014;30(11):944-52.

24. Sarakatsianou C, Theodorou E, Georgopoulou S, Stamatiou G, Tzovaras G. Effect of pre-emptive pregabalin on pain intensity and postoperative morphine consumption after laparoscopic cholecystectomy. Surg Endosc. 2013;27(7):250411.

25. Balaban F, Yagar S, Özgök A, Koç M, Güllapoglu $H$. A randomized, placebo-controlled study of pregabalin for postoperative pain intensity after laparoscopic cholecystectomy. J Clin Anesth. 2012;24(3):175-8.

26. Dereli N, Tutal ZB, Babayigit M, Kurtay A, Sahap $M$, Horasanli E. Effect of intraoperative esmolol infusion on anesthetic, analgesic requirements and postoperative nausea-vomitting in a group of laparoscopic cholecystectomy patients. Rev Bras Anestesiol. 2015;65(2):141-6. Portuguese.

27. Delfino $A E$, de la Fuente $N$, Echevarría GC, Altermatt FR, Cortinez LI. Effect of acute arterial hypertension on morphine requirements and postsurgical pain. J Clin Anesth. 2015;27(3):226-32.

28. Akelma FK, Ergil J, Özkan D, Akinci M, Özmen $M$, Gümüs J. A comparison of the effects of intraoperative esmolol and lidocaine infusions on postoperative analgesia. Anestezi Dergisi. 2014;22(1):25-31.

29. Lee JH, Kim JH, Cheong YK. The analgesic effect of nefopam with fentanyl at the end of laparoscopic cholecystectomy. Korean J Pain. 2013;26(4):361-7.

30. Park JK, Cheong SH, Lee KM, Lim SH, Lee JH, Cho K, et al. Does dexmedetomidine reduce postoperative pain after laparoscopic cholecystectomy with multimodal analgesia? Korean J Anesthesiol. 2012;63(5):436-40 
31. López-Álvarez S, Mayo-Moldes $M$, Zaballos $M$, Iglesias BG, Blanco-Dávila R. Esmolol versus ketamine-remifentanil combination for early postoperative analgesia after laparoscopic cholecystectomy: a randomized controlled trial. Can J Anaesth. 2012;59(5):442-8.

32. Elamin G, Waters PS, Hamid H, O'Keeffe HM, Waldron RM, Duggan $M$, et al. Efficacy of a laparoscopically delivered transversus abdominis plane block technique during elective laparoscopic cholecystectomy: a prospective, double-blind randomized trial. J Am Coll Surg. 2015;221(2):33544.

33. Basaran B, Basaran A, Kozanhan B, Kasdogan E, Eryilmaz MA, Ozmen S. Analgesia and respiratory function after laparoscopic cholecystectomy in patients receiving ultrasound-guided bilateral oblique subcostal transversus abdominis plane block: a randomized double-blind study. Med Sci Monit. 2015;21:1304-12.

34. Pandey A, Kumar R, Kang CLS, Agarwal M. Comparative study between levobupivacaine with clonidine and ropivacaine with clonidine in thoracic epidural block for laparoscopic cholecystectomy. J Evolution Med Dent Sci. 2015;4(9):1457-65.

35. Bhatia N, Arora S, Jyotsna W, Kaur G. Comparison of posterior and subcostal approach to ultrasound-guided transverse abdominis plane block for postoperative analgesia in laparoscopic cholecystectomy. J Clin Anesth. 2014;26(4):294-9.

36. Petersen $\mathrm{PL}$, Stjernholm $\mathrm{P}$, Kristiansen $\mathrm{VB}$, Torup $\mathrm{H}$, Hansen EG, Mitchell AU, et al. The beneficial effect of transversus abdominis plane block after laparoscopic cholecystectomy in day-case surgery: a randomized clinical trial. Anesth Analg. 2012;115(3):527-33.

37. Choi YJ, Park SW, Kwon HJ, Choi JM, Lee YM. Efficacy of early intravenous bolus oxycodone or fentanyl in emergence from general anaesthesia and postoperative analgesia following laparoscopic cholecystectomy: a randomized trial. J Int Med Res. 2015;43(6):809-18.

38. Hwang BY, Kwon JY, Kim E, Lee DW, Kim TK, Kim HK. Oxycodone vs. fentanyl patient-controlled analgesia after laparoscopic cholecystectomy. Int J Med Sci. 2014;11(7):658-62.

Recebido em: 02/04/2018

Aceito para publicação em: 17/05/2018

Conflito de interesse: nenhum.

Fonte de financiamento: nenhuma.

Endereço para correspondência: Renato Ribeiro de Jesus E-mail: renatordejesus@gmail.com ok.renato@hotmail.com 